\title{
Evaluating the Learning Object Metadata for K-12 Educational Resources
}

\author{
Daniel D. Suthers \\ Department of Information and Computer Sciences \\ University of Hawai i at Manoa \\ suthers@hawaii.edu
}

\begin{abstract}
The Learning Object Metadata (LOM) is an emerging standard for organizing descriptions of digital or nondigital entities used to support learning. The descriptions include educational, legal, and technical characteristics of these resources. In this paper we describe an application of the LOM to the construction of a database of resources available to public schools in Hawai $i$ and report on issues encountered, focusing on structural issues such as dependencies between elements. The paper illustrates why development of metadata formats cannot be divorced from an understanding of educational context.
\end{abstract}

\section{Introduction}

Internet technology for learning has the potential to bring teachers and students together with a greater diversity of human, natural and technological resources than was previously possible. Additionally, the current emphasis on systemic reform in public school education in the United States is encouraging a greater diversity of stakeholders to collaborate in supporting students' achievement of high standards. These forces require that educators and their partners be aware of the resources that are potentially available to them and to understand the utility of these resources with respect to educational objectives. Already pressed for time, how will educators sort through this cornucopia of information and misinformation and find the resources appropriate for the educational needs of their students? Clearly, educators will need help. This paper is concerned with one form of help: databases of metadata [5] or information that describes the relevant characteristics of educational resources sometimes called learning objects. Properly constructed metadata should enable educators to find relevant learning objects more quickly. Examples of metadata databases include ARIADNE [1], NEEDS [6], and PEN-DOR [2].

Resource databases should adequately describe a diverse variety of resources yet relate them to educational objectives, describe the resources in terms understandable to educators, and interoperate with other major repositories. In this paper I report on our evaluation of an emerging standard, the Learning Object Metadata (LOM) with respect to its suitability for describing K-12 resources as part of a systemic initiative known as Hawai i Networked Learning Communities. Specifically I discuss limitations and extensions to the LOM that were required, focusing on structural issues.

\section{The LOM}

The Learning Technology Standards Committee (LTSC), founded in 1996 by a group of academic, government, and industry representatives (including the author), is an umbrella organization that sponsors approximately 15 learning technology standards efforts, under the sponsorship of the IEEE (Institute of Electrical and Electronics Engineers, http://www.ieee.org/). The LOM draft standard (also known by its IEEE identifier as 1484.12) is arguably the most mature of the LTSC draft standards. According to draft 6 of the LOM [4], "The purpose of this standard is to facilitate search, evaluation, acquisition, and use of learning objects, for instance by learners or instructors. The purpose is also to facilitate the sharing and exchange of learning objects, by enabling the development of catalogs and inventories while taking into account the diversity of cultural and lingual contexts in which the learning objects and their metadata will be exploited."

The LOM standard is meant to provide a semantic model for describing properties of the learning objects themselves, rather than detailing ways in which these learning objects may be used to support learning. The LOM indicates the legal values and informal semantics of the metadata elements, their dependencies on each other, and how they are composed into a larger structure. It is intended to be extended, and in fact a structure has been provided specifically for the purpose. The LOM information structures are intended to support information exchange, and are neither specifications of an implementation nor specifications of a user interface. The LOM is agnostic concerning bindings or implementations of metadata in representations or notations. 
The LOM metadata elements as of draft 6 [4] includes the following major element categories. 1:General provides information such as title, a brief textual description, and keywords. 2:Life.Cycle describes the development and current state of the learning object. 3:Metameta.Data describes the metadata itself, e.g., who entered or validated this metadata instance and what language it is written in. 4:Technical provides information on media type, size, software requirements, etc. for those learning objects to which these attributes apply. 5:Educational is intended to provide basic information about the pedagogical characteristics of the learning object. This category includes some of the most controversial elements, to be discussed further below. 6:Rights describes the conditions under which one may acquire and use the learning object. 7:Relation can be used to describe the learning object in relation to other learning objects. 8:Annotation records comments on the educational use of the learning object. 9:Classification provides a means of extending the LOM to meet specialized needs.

\section{HNLC Resource Database}

The Hawai' $i$ Networked Learning Communities (HNLC, http://lilt.ics.hawaii.edu/hnlc/) initiative is a partnership between the Hawai i Department of Education (HDOE), the University of Hawai i, and many other stakeholders in the quality of Hawai i public education, such as business and nonprofit interests. HNLC's purpose is to prepare all students in Hawaii's public schools for careers in today's technological world by enabling them to attain high standards in science, math, and technology education. One component of the work of HNLC includes development of a web-accessible database with which educators can find and discuss educational resources available in Hawai'i. This paper analyzes the suitability of the LOM for this database.

The database describes resources for public school education ranging from US grades Kindergarten $(\mathrm{K})$ to 12 , abbreviated as $\mathrm{K}-12$. A wide variety of digital and nondigital resources will be described, making this a particularly challenging test implementation of the LOM. To control the scope of our work, HNLC will prioritize the description of local resources and interface with other repositories of nationally available resources such the Gateway to Educational Materials (GEM, [7]).

Our method of evaluation was as follows. Initially we wrote informal textual descriptions capturing the important information about a representative sample of the resources that we wanted to describe. After reviewing these descriptions I presented the then-current LOM draft 4.1 [3] to the entire team. We then went through the textual descriptions and identified LOM elements in which the information expressed could be captured. Where we failed to find LOM elements for an item of information, we extended the LOM, either by expanding on the vocabulary of an existing element or by creating an entirely new element under 9:Classification. Where new elements were needed we searched other repositories to find metadata that we could use. Several iterations were required to understand the LOM structure well enough to define our instances of 9:Classification. Then our programmer created a Filemaker implementation of the resulting HNLC-LOM and provided the others with an interface for building metadata. Metadata for our sample was then created by two team members, and I reviewed the result to detect possible misunderstandings and issues. I also compiled a first draft of issues and recommendations. This draft was shared with the LTSC LOM committee, both via email and subsequently face to face in an LTSC meeting (Montreal, June 2000). Thanks to their feedback, many issues were resolved or reunderstood as non-issues.

\section{Vocabulary Issues}

The data type of draft 4.1 LOM elements were either primitive (e.g., a string), referenced other standards (e.g., vCard), or consisted of a controlled vocabulary [3]. In the latter case, the vocabulary was either restricted, meaning that only the terms listed may be used, or open with recommended practice, meaning that one should attempt to use one of the terms listed as the recommended practice but may extend this vocabulary if needed. Draft 4.1 required that one accomplish an extension of a vocabulary with term term by placing a tuple of form ("See_Classification", term) in the data element, and defining an instance of 9:Classification that has the same 9.1:Purpose as the data element being extended. This 9:Classification instance would include one or more instances of 9.2:Taxon.Path as needed to indicate where the term falls within the taxonomic system indicated by sub-element 9.2.1:Source. See Figure 1 (draft 4.1) for an example. A taxon path can be thought of as a sequence of taxons, which begins at the root of a taxonomic hierarchy and works its way down the tree through intermediate nodes to the leaf node under which the object is being classified.

This arrangement provided a powerful generalpurpose way of extending vocabularies with information about the taxonomic source of the term, and hence its semantics. However, subsequent to our evaluation, See_Classification was judged to be too complex, and removed in favor of a simpler system in which all vocabulary items have form (Source, Value). Source 


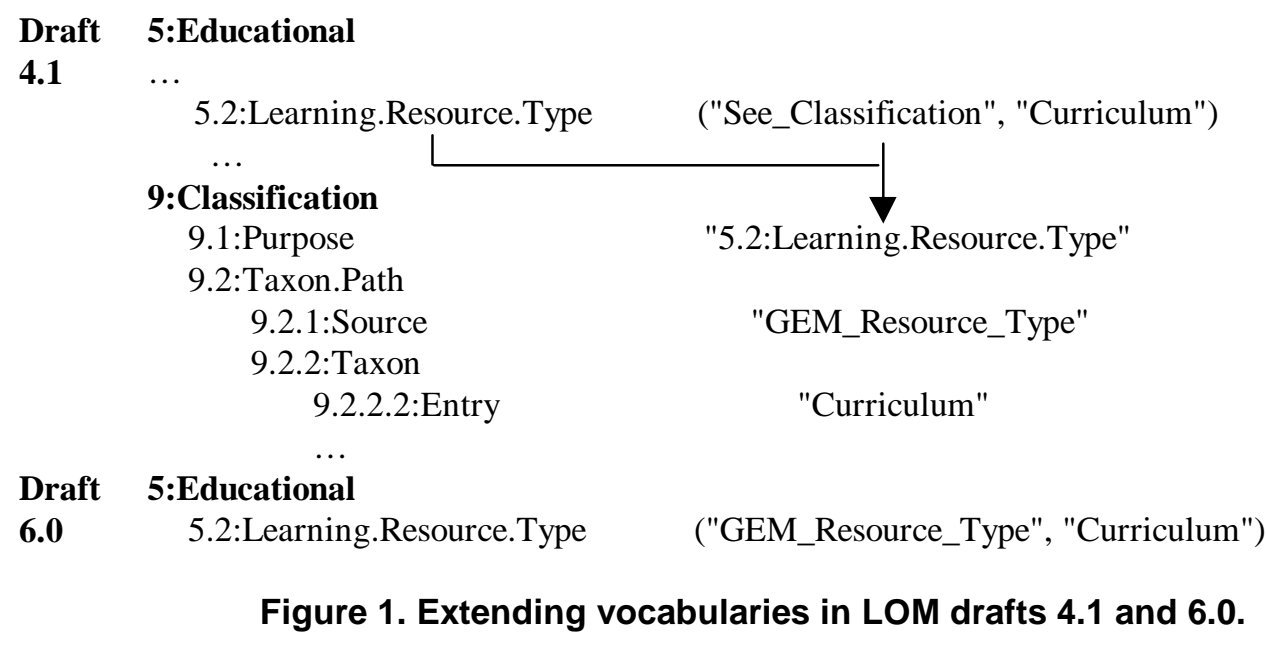

would either be "LOMv1.0" or an indication of the alternative source of the value, as illustrated in Figure 1 (draft 6.0). The example is simplified: Uniform Resource Identifiers would be used to identify the Source.

We found several of the LOM vocabularies to be insufficient for our purposes. In one case, 5.2:Learning Resource Type, the vocabulary was open and the insufficiencies could be addressed via the extension mechanism just described. However, vocabularies for 1.9:Aggregation.Level, 5.1:Interactivity.Type (values: Active, Expositive. Mixed, or Undefined) and 5.5:Intended.End.User.Role (Teacher, Author, Learner, Manager) were restricted vocabularies, so could not be extended in this way. Subsequent to our report to the LTSC, these vocabularies and all others have been opened: there are no restricted vocabularies. This is an important recognition of the preliminary nature of vocabularies chosen by committee and the importance of allowing user communities to derive useful vocabularies.

\section{Structural Issues}

In some cases, including 5.1:Interactivity.Type, 5.5:Intended.End.User.Role (see next section), and 5.7:Typical.Age.Range, we felt that the vocabulary should be replaced with a structured description. Concerning 5.7:Typical.Age.Range, K-12 educational resources in the United States are often referenced by grade level rather than age range. Other applications may require other measures, for example, the military needs to access resources by rank. Therefore we recommended that 5.7:Typical.Age.Range be renamed and changed to a structured element with 5.7.1 Measure (e.g., "Chronological Age," "GEM Grade," etc.) and 5.7.2:Value (e.g., "12," "7-8," etc.).
More problematic are ways in which the value of one data element depends on another. We noted that 5.9:Typical.Learning. Time depends on the value of 5.7:Typical.Age.Range, for example, a textbook might be described as "suitable for a fast paced graduate course or a two-semester undergraduate sequence." Erik Duval (technical editor for the LOM) later pointed out that this applies to 5.4:Semantic.Density and 5.8:Difficulty as well. Hence we recommended reorganizing this information in the following manner:

5.x:Challenge Level consisting of one or more 4-tuples: 5.x.1:Educational Level (formerly 5.7): 5.x.1.1:Measure (e.g., Age, US Grade)

5.x.1.2:Value (e.g., 7-8)

5.x.2:Semantic Density (formerly 5.4)

5.x.3:Difficulty (formerly 5.8)

5.x.4:Learning Time (formerly 5.9)

Then one could create multiple instances of 5.x:Challenge.Level, with the values of 5.x.2 through 5.x.4 being dependent on the value of 5.x.1:Educational.Level. It is possible to implicitly achieve the same effect by replicating entire LOM metadata instances, one for each developmental level (or age); but it is far more perspicuous and efficient to acknowledge the dependency explicitly in a structure such as that above. The proposals of this section have not been adopted at this writing.

\section{Our Extensions to the LOM}

The following extensions were made using 9:Classification. Some will be replaced with the draft 6.0 structured vocabulary items. 
We replaced 5.5:Intended.End.User.Role with the GEM Audience 1 a two-part classification consisting of ToolFor (who uses the tool) and Beneficiary (who benefits). For example, a professional development resource that helps teachers handle learning-disabled children in their classes is for the teacher but benefits the particular population of learning disabled students. Using LOM draft 6.0 we could write (GEM_Tool_For, value) and (GEM_Beneficiary, value).

Discipline describes the subject matter area covered by the resource. The LOM intentionally leaves this for 9:Classification. We are using the GEM Subject, originally designed to be a simple pointer to a subject area, with provisions for subject terms added from other controlled vocabularies [8]. This is a two-level classification system, requiring a two-step Taxon Path, for example Science/Astronomy.

Educational Objective addresses content and performance standards, specifically the Hawai' i Content and Performance Standards in our application. It is distinct from Discipline because it is more specific: it aligns the resource with the particular educational standards that the resource is intended to help achieve.

Educational Level augments LOM 5.7:Typical.Age.Range, and is structured as described in the previous section, using Source for Measure and Taxon for Value. The current 9:Classification is not sufficiently expressive to achieve the more complex 5.x:Challenge.Level we proposed.

We designed a classification called Pedagogy to replace 5.1:Interactivity.Type, which has an extremely deficient vocabulary of active, expositive, mixed, undefined and was restricted in draft 4.1 A rich description of interactivity is available in the GEM Pedagogy controlled vocabulary. This vocabulary has three facets: Teaching Methods (GEM provides a large vocabulary), Grouping (individual, small group, large group, etc.), and Assessment. Subsequently, 5.1:Interactivity. Type has been unrestricted.

\section{Conclusions}

In this paper I described our attempt to use the LOM for a K-12 resource database. We found that it provides a solid foundation in the form of many well thought-out data elements as well as a means for extension. We also found that the LOM does not address all the needs of our application. This is not surprising, as the LOM is being designed to serve a variety of applications in government and industry as well as public education. We were able to

\footnotetext{
${ }^{1}$ See http://www.geminfo.org/Workbench/Metadata/ for all GEM Metadata described here.
}

deal with most of the limitations through the Classification method of extension. Some of these solutions would be converted to structured vocabulary items under LOM draft 6.0. However, there remain some structural dependencies between LOM elements that are not well captured. These issues were illustrated with examples from K-12 education. It is hoped that this paper will help increase awareness within the primary/secondary education sector of the LOM standards effort, and encourage this sector's contribution to further development of the standard to be more appropriate for primary/secondary education needs. Further information may be obtained at http://ltsc.ieee.org/wg12/index.html.

\section{Acknowledgements}

Thanks to Beth Tillinghast and Susan Johnson for for contributing to the design of the HNLC metadata and constructing metadata, David Nickles for implementing the first prototype of our resource database, and Laura Girardeau for editorial assistance. Thanks to Erik Duval, Wayne Hodgins, Tom Murray, Brendon Towle, and Tom Wason for their "metacomments" on my LOM commentary. This work was funded by a development grant from the National Science Foundation's Rural Systemic Initiative.

\section{References}

[1] Forte, E., Haenni, F., Warkentyne, K., Duval, E., Cardinaels, K., Vervaet, E., Hendrikx, K., Forte, M. W., \& Simillion, F. (1999). Semantic and Pedagogic interoperability mechanism in the ARIADNE educational repository, SIGMOD Record, 28(1), 20-25.

[2] Fullerton, K, Greenberg, J., McClure, M., Rasumussen, E., \& Stewart, D. (1999). A digital library for education: the PEN-DOR project. The Electronic Library, 17 (2), 75-82.

[3] IEEE Std. 1484.12 (draft). Draft 4.1 of the Learning Objects Metadata (LOM), IEEE, Piscataway, N.J., USA, February 5, 2000.

[4] IEEE Std. 1484.12 (draft). Draft 6.0 of the Learning Objects Metadata (LOM), IEEE, Piscataway, N.J., USA, February 8, 2001. Available: http://ltsc.ieee.org/wg12/index.html

[5] Milstead, J. \& Feldman, S. (1999). Metadata: Cataloging by any other name. Online 23(1),24-31.

[6] Muramatsu, B. \& Agogino, A. (1999). The National Engineering Education Delivery System: A digital library for engineering education. D-Lib Magazine 5(4).

[7] Sutton, S. (1998). Gateway to Educational Materials (GEM): Metadata for networked information discovery and retrieval. Computer Networks and ISDN Systems 30(1-7), 91-93.

[8] Sutton, S. (1999). Conceptual design and deployment of a metadata framework for educational resources on the Internet. Journal of the American Society for Information Science 50(13), 1182-92. 